Acta Crystallographica Section E

Structure Reports

Online

ISSN 1600-5368

S. A. Khanum, ${ }^{\text {a }}$ M. Mahendra, ${ }^{b}$ S. Shashikanth, ${ }^{\mathrm{a}}$ B. H. Doreswamy, ${ }^{b}$ M. A. Sridhar ${ }^{b}$ and J. Shashidhara Prasad ${ }^{\mathrm{b} *}$

aDepartment of Studies in Chemistry, Mansagangotri, University of Mysore, Mysore 570 006, India, and ${ }^{\mathbf{b}}$ Department of Studies in Physics, Mansagangotri, University of Mysore, Mysore 570 006, India

Correspondence e-mail:

mas@physics.uni-mysore.ac.in

\section{Key indicators}

Single-crystal X-ray study

$T=295 \mathrm{~K}$

Mean $\sigma(\mathrm{C}-\mathrm{C})=0.004 \AA$

$R$ factor $=0.049$

$w R$ factor $=0.151$

Data-to-parameter ratio $=12.5$

For details of how these key indicators were automatically derived from the article, see http://journals.iucr.org/e.

\title{
(3-Chlorophenyl)(2-hydroxy-5-methylphenyl)- methanone
}

In the title compound, $\mathrm{C}_{15} \mathrm{H}_{14} \mathrm{ClO}_{2}$, the dihedral angle between the two benzene rings is $57.37(12)^{\circ}$.

\section{Comment}

The significance of benzophenone analogues in biological systems, as well as in chemotherapy, is now well established (Hsieh et al., 2003; Revesz et al., 2004). The chemistry of hydroxybenzophenones constitutes a central and important area of interest in synthetic organic, medicinal and pharmacological chemistry (Cuesta-Rubio et al., 2002; Schlitzer et al., 2002; Vidya et al., 2003). Serving as attractive scaffolds for drug design and conferring drug-like characteristics on numerous structural motifs, halo-substituted hydroxybenzophenones are finding increasing applications in organic and medicinal chemistry (Khanum et al., 2005). Based on the above observations, the title compound, (I), was synthesized and its crystal structure is reported here.<smiles>Cc1ccc(O)c(C(=O)c2cccc(Cl)c2)c1</smiles>

(I)

The molecule of (I) is non-planar (Fig. 1). The dihedral angle between the two benzene rings is $57.37(12)^{\circ}$, a value much smaller than that of $75.2^{\circ}$ observed for (2-chlorophenyl)(3,4-dimethoxyphenyl)methanone, (II) (Mahendra et al., 2003). The bond lengths and angles have normal values and are comparable with those reported for (II). The crystal packing is stabilized by intramolecular O9-H9..O12 and intermolecular C6-H6...O12 hydrogen bonds (Table 2), which link the molecules into chains (Fig. 2). A detailed study of the biological activity of (I) is underway.

\section{Experimental}

A solution of anhydrous aluminium chloride $(3.2 \mathrm{~g}, 0.02 \mathrm{~mol})$ in dry nitrobenzene $(25 \mathrm{ml})$ was added to 4-methylphenyl chlorobenzoate $(5 \mathrm{~g}, 0.02 \mathrm{~mol})$ dissolved in nitrobenzene $(10 \mathrm{ml})$. The mixture was protected from moisture by a calcium chloride guard tube and refluxed with stirring for $30 \mathrm{~min}$. At the end of this period, the solution was cooled and treated with acidic ice-cold water. Nitro-
Received 3 October 2005 Accepted 5 October 2005 Online 12 October 2005 
benzene was removed by steam distillation. The residual solid was crushed into a powder, extracted with $10 \%$ sodium hydroxide $(150 \mathrm{ml})$, and the basic aqueous solution was neutralized with $10 \%$ hydrochloric acid. The product was extracted into diethyl ether and the ether layer washed well with a saturated sodium chloride solution. Evaporation of the ether after drying over anhydrous sodium sulfate followed by recrystallization from methanol gave (I) in $85 \%$ yield (m.p. 344-346 K). IR (Nujol): $1673(\mathrm{C}=\mathrm{O}), 3550-3640 \mathrm{~cm}^{-1}(\mathrm{OH})$; ${ }^{1} \mathrm{H}$ NMR $\left(\mathrm{CDCl}_{3}\right): 2.2\left(s, 3 \mathrm{H}, \mathrm{CH}_{3}\right), 7.0-7.65(m, 7 \mathrm{H}, \mathrm{Ar}-\mathrm{H}), 12.15$ (bs, 1H, OH); MS (EI) $m / z: 246\left(M^{+}, 88\right)$; Analysis calculated for $\mathrm{C}_{14} \mathrm{H}_{11} \mathrm{ClO}_{2}$ : C 68.15, $\mathrm{H} 4.46, \mathrm{Cl} 14.40 \%$; found: $\mathrm{C} 68.17, \mathrm{H} 4.44, \mathrm{Cl}$ $14.42 \%$.

\section{Crystal data}

$\mathrm{C}_{14} \mathrm{H}_{11} \mathrm{ClO}_{2}$

$M_{r}=246.68$

Monoclinic, $P 2_{1} / c$

$a=10.485$ (9) А

$b=7.823$ (4) $\AA$

$c=16.297(13) \AA$

$\beta=116.949(2)^{\circ}$

$V=1191.59(15) \AA^{3}$

$Z=4$

$$
\begin{aligned}
& D_{x}=1.375 \mathrm{Mg} \mathrm{m}^{-3} \\
& \text { Mo } K \alpha \text { radiation } \\
& \text { Cell parameters from } 3643 \\
& \quad \text { reflections } \\
& \theta=2.3-25.0^{\circ} \\
& \mu=0.31 \mathrm{~mm}^{-1} \\
& T=295(2) \mathrm{K} \\
& \text { Block, pale yellow } \\
& 0.3 \times 0.2 \times 0.2 \mathrm{~mm}
\end{aligned}
$$

\section{Data collection}

MacScience DIPLabo 32001 diffractometer

$\omega$ scans

Absorption correction: none

3643 measured reflections

1945 independent reflections

$$
\begin{aligned}
& 1690 \text { reflections with } I>2 \sigma(I) \\
& R_{\text {int }}=0.017 \\
& \theta_{\max }=25.0^{\circ} \\
& h=-12 \rightarrow 12 \\
& k=-8 \rightarrow 7 \\
& l=-19 \rightarrow 19
\end{aligned}
$$

\section{Refinement}

Refinement on $F^{2}$

$R\left[F^{2}>2 \sigma\left(F^{2}\right)\right]=0.049$

$w R\left(F^{2}\right)=0.151$

$S=1.09$

1945 reflections

156 parameters

$\mathrm{H}$-atom parameters constrained

$$
\begin{aligned}
& w=1 /\left[\sigma^{2}\left(F_{\mathrm{o}}^{2}\right)+(0.089 P)^{2}\right. \\
& \quad+0.3587 P] \\
& \text { where } P=\left(F_{\mathrm{o}}^{2}+2 F_{\mathrm{c}}^{2}\right) / 3 \\
& (\Delta / \sigma)_{\max }<0.001 \\
& \Delta \rho_{\max }=0.56 \mathrm{e}^{-3} \\
& \Delta \rho_{\min }=-0.33 \mathrm{e}^{-3} \\
& \text { Extinction correction: SHELXL97 } \\
& \text { Extinction coefficient: } 0.069(8)
\end{aligned}
$$

Table 1

Selected geometric parameters $\left(\AA{ }^{\circ}\right)$.

\begin{tabular}{llll}
\hline $\mathrm{C} 11-\mathrm{C} 15$ & $1.747(3)$ & $\mathrm{O} 12-\mathrm{C} 11$ & $1.234(3)$ \\
$\mathrm{O} 9-\mathrm{C} 8$ & $1.351(3)$ & & \\
$\mathrm{O} 9-\mathrm{C} 8-\mathrm{C} 7$ & $117.9(2)$ & $\mathrm{O} 12-\mathrm{C} 11-\mathrm{C} 10$ & $121.2(2)$ \\
$\mathrm{O} 9-\mathrm{C} 8-\mathrm{C} 10$ & $123.05(19)$ & $\mathrm{Cl} 1-\mathrm{C} 15-\mathrm{C} 16$ & $119.5(3)$ \\
$\mathrm{O} 12-\mathrm{C} 11-\mathrm{C} 13$ & $118.0(2)$ & $\mathrm{Cl} 1-\mathrm{C} 15-\mathrm{C} 14$ & $118.72(19)$ \\
\hline
\end{tabular}

Table 2

Hydrogen-bond geometry $\left(\AA,^{\circ}\right)$.

\begin{tabular}{lllll}
\hline$D-\mathrm{H} \cdots A$ & $D-\mathrm{H}$ & $\mathrm{H} \cdots A$ & $D \cdots A$ & $D-\mathrm{H} \cdots A$ \\
\hline O9-H9 $\cdots \mathrm{O} 12$ & 0.82 & 1.87 & $2.585(3)$ & 145 \\
C6-H6 $\cdots$ O12 & 0.93 & 2.54 & $3.408(3)$ & 156
\end{tabular}

Symmetry code: (i) $x,-y+\frac{3}{2},+z-\frac{1}{2}$.

Difficulties with processing some strong reflections led to their omission from the data set, limiting the completeness of data used in this determination. $\mathrm{H}$ atoms were placed at idealized positions and allowed to ride on their parent atoms, with $\mathrm{C}-\mathrm{H}$ distances of $0.96 \AA$ and $U_{\text {iso }}(\mathrm{H})$ values set equal to $x U_{\text {eq }}$ (carrier atom), where $x=1.5$ for

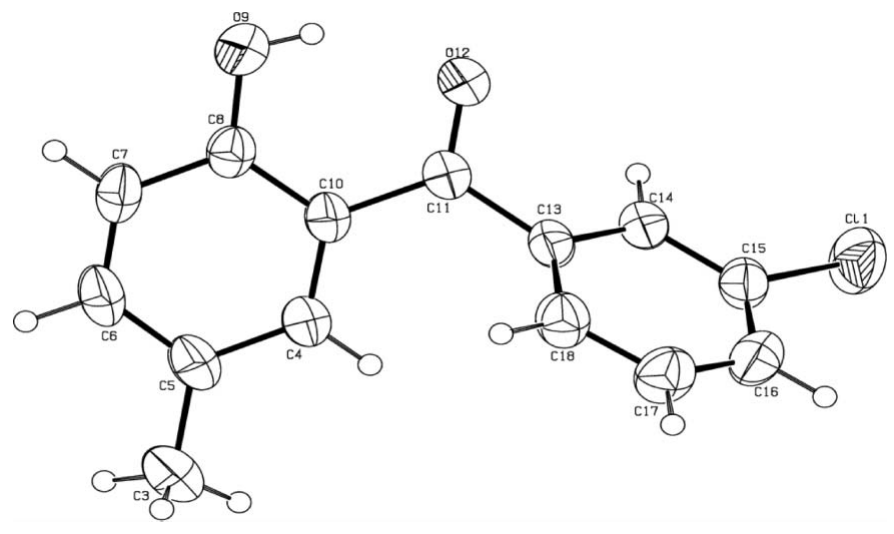

Figure 1

View of (I), shown with $50 \%$ probability displacement ellipsoids.

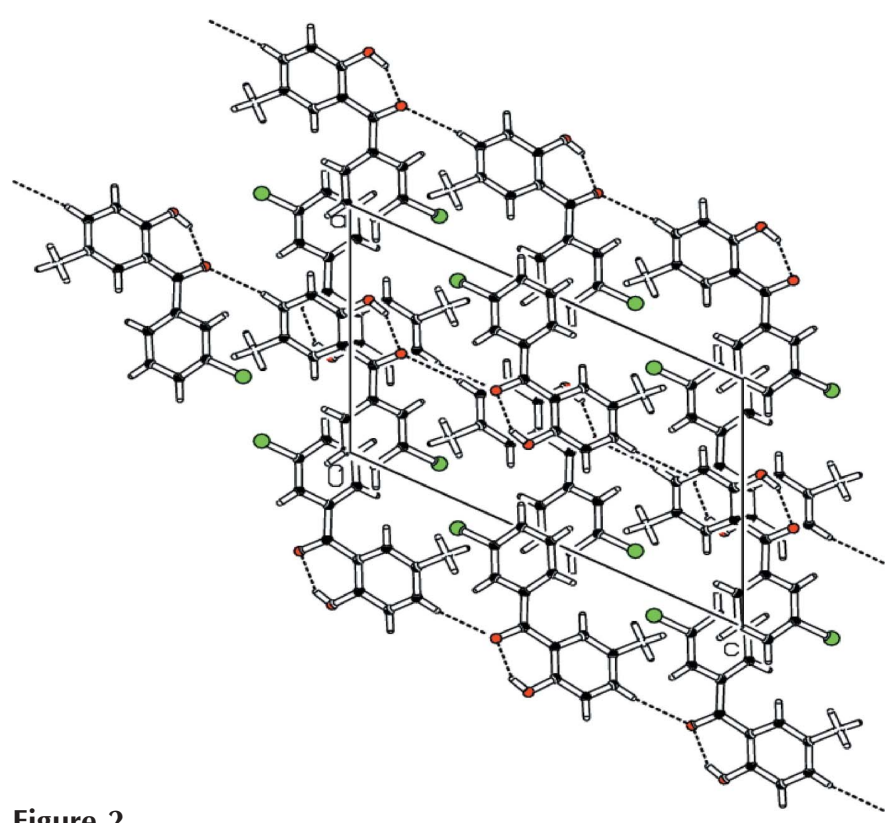

Figure 2

The crystal packing in (I), viewed down the $b$ axis. Dashed lines indicate hydrogen bonds.

methyl and hydroxyl $\mathrm{H}$ atoms and 1.2 for other $\mathrm{H}$ atoms. A rotating group refinement was used for the methyl groups.

Data collection: XPRESS (MacScience, 2002); cell refinement: SCALEPACK (Otwinowski \& Minor, 1997); data reduction: DENZO (Otwinowski and Minor, 1997) and SCALEPACK; program(s) used to solve structure: SHELXS97 (Sheldrick, 1990); program(s) used to refine structure: SHELXL97 (Sheldrick, 1997); molecular graphics: PLATON (Spek, 2003) and ORTEPII (Johnson, 1976); software used to prepare material for publication: SHELXL97.

The authors thank the DST, Government of India, for financial assistance under project No. SP/I2/FOO/93. MM thanks the CSIR, Government of India, for the award of a Senior Research Fellowship.

\section{References}

Cuesta-Rubio, O., Frontana-Uribe, B. A., Ramirez-Apan, T. \& Cardenas, J. (2002). J. Biosci. 57, 372-378. 
Hsieh, H. P., Liou, J. P., Lin, Y. T., Mahindroo, N., Chang, J. Y., Yang, Y. N., Chern, S. S., Tan, U. K., Chang, C. W., Chen, T. W., Lin, C. H., Chang, Y. Y. \& Wang, C. C. (2003). Bioorg. Med. Chem. Lett. 13, 101105.

Johnson, C. K. (1976). ORTEPII. Report ORNL-5138. Oak Ridge National Laboratory, Tennessee, USA.

Khanum, S. A., Satish, K. M., Vishwanth, B. S. \& Shashikanth, S. (2005). Bioorg. Med. Chem. Lett. 15, 4100-4104.

MacScience (2002). XPRESS. MacScience Co. Ltd, Yokohama, Japan.

Mahendra, M., Doreswamy, B. H., Sudha, B. S., Khanum, S. A., Shashikanth, S., Sridhar, M. A. \& Prasad, J. S. (2003). J. Anal. Sci. 19, x57$\mathrm{x} 58$.
Otwinowski, Z. \& Minor, W. (1997). Methods in Enzymology, Vol. 276, Macromolecular Crystallography, Part A, edited by C. W. Carter Jr \& R. M. Sweet, pp. 307-326. New York: Academic Press.

Revesz, L., Blum, F. E., Di Padova, E. T., Buhl, R., Feifel, H., Gram, P., Hiestand, U., Manning, A. \& Rucklin, G. (2004). Bioorg. Med. Chem. Lett. 14, 3601-3605..

Schlitzer, M., Bohm, M. \& Sattler, I. (2002). Bioorg. Med. Chem. 10, 615-620. Sheldrick, G. M. (1990). Acta Cryst. A46, 467-473.

Sheldrick, G. M. (1997). SHELXL97. University of Göttingen, Germany. Spek, A. L. (2003). J. Appl. Cryst. 36, 7-13.

Vidya, R., Eggen, M., Georg, G. I. \& Himes, R. H. (2003). Bioorg. Med. Chem. Lett. 13, 757-760. 